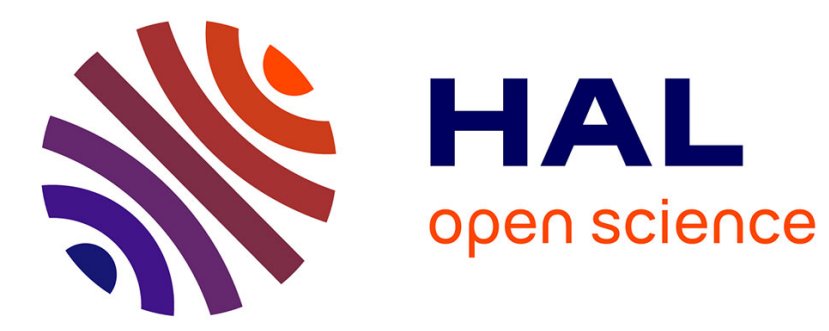

\title{
Photothermally modulated ferromagnetic resonance investigations of epitaxially grown thin Fe (001) films
}

\author{
R. Meckenstock, O. von Geisau, F. Schreiber, E. da Silva, J. Pelzl
}

\section{To cite this version:}

R. Meckenstock, O. von Geisau, F. Schreiber, E. da Silva, J. Pelzl. Photothermally modulated ferromagnetic resonance investigations of epitaxially grown thin Fe (001) films. Journal de Physique IV Proceedings, 1994, 04 (C7), pp.C7-663-C7-666. 10.1051/jp4:19947157 . jpa-00253216

\section{HAL Id: jpa-00253216 https://hal.science/jpa-00253216}

Submitted on 1 Jan 1994

HAL is a multi-disciplinary open access archive for the deposit and dissemination of scientific research documents, whether they are published or not. The documents may come from teaching and research institutions in France or abroad, or from public or private research centers.
L'archive ouverte pluridisciplinaire HAL, est destinée au dépôt et à la diffusion de documents scientifiques de niveau recherche, publiés ou non, émanant des établissements d'enseignement et de recherche français ou étrangers, des laboratoires publics ou privés. 


\title{
Photothermally modulated ferromagnetic resonance investigations of epitaxially grown thin Fe (001) films
}

\author{
R. Meckenstock, O. von Geisau, F. Schreiber, E.C. da Silva* and J. Pelzl \\ Fakultät für Physik und Astronomie, AG Festkörperspektroskopie, Ruhr-Universität Bochum, \\ 44780 Bochum, Germany \\ * Instituto de Fisica, Universidade Estadual de Campinas, 13081 Campinas, Sao Paulo, Brazil
}

\begin{abstract}
The photothermally modulated (PM) ferromagnetic resonance (FMR) combines the high sensitivity of conventional FMR and the spatial resolution of thermal waves. For the first time, this alternative FMR-technique was applied to the magnetic characterization of high-quality thin Fe (001) films epitaxially grown on (001) GaAs substrate: Images of the distribution of crystalline anisotropy constant $K_{I}$ and the magnetization $M$ were determined from the locally resolved FMR spectra.
\end{abstract}

\section{INTRODUCTION}

Thin metallic films and superlattices have become one of the major fields of research in magnetism. Molecular beam epitaxy offers the opportunity to design magnetic systems with new properties being of interest for both applied and basic research [1,2]. The magnetic behaviour of the films above all depends on the magnetic anisotropy and the exchange interaction which can be investigated very precisely by the conventional ferromagnetic resonance (FMR) technique $[3,4]$. The FMR measures the precession amplitude of the magnetization $M$ in an effective internal magnetic field $B_{\text {eff }}$. This field is composed of the external magnetic field $B_{0}$, the demagnetizing field and different anisotropy fields. A semiclassical description of FMR is given by the Landau-Lifshitz equation of motion for $M$ [5]. Most frequently, in FMR measurements, the magnetic field value $B_{0}$ of the resonance line is studied as a function of the external field orientation. In addition, other parameters such as the width, the shape or the intensity of the resonance line contain some relevant information. Broad line widths or strongly asymmetric line shapes can often be taken as indication of inhomogeneities or considerable strains in the films. However, the conventional FMR only yields information on the absorption of the incident microwave power integrated over the sample volume. The lack of spatial resolution can be overcome by the photothermally modulated (PM-) FMR. Here, the field modulation of the conventional FMR detection is replaced by a temperature modulation caused by an intensity modulated laser beam directed onto the sample [6-8]. The resonance absorption of microwaves in general depends on the sample temperature $T$. Thus, the temperature oscillations due to the periodical heating with the laser beam give rise to an $a c$-contribution to the absorbed microwave power which can be detected synchronously. Lateral resolution is achieved by focusing the laser and scanning the sample. A depth resolution is obtained by making use of the modulation frequency dependent changes in the thermal diffusion length, i. e. the penetration depth of the thermal waves generated in the sample.

The possibility of obtaining spatially resolved information with PM-FMR has been used to study various systems such as magnetic tapes, polycrystalline metallic films $[9,7]$, and magnetostatic modes in yttrium iron garnet $[10,8,11]$. 
In this paper, we report on the application of the PM-FMR to single crystalline metallic thin films of Fe $(20 \mathrm{~nm})$ grown on a GaAs substrate. The signal generation process is discussed and it will be shown that the PM-FMR can be used to obtain images of magnetic properties of epitaxial films such as crystalline anisotropy constant $K_{l}$ and magnetization $M$. Besides the magnetic properties of the sample, the experiment also yields information on the electronic properties of the semiconducting substrate material [11].

\section{EXPERIMENT}

The experimental setup consists of a modified conventional FMR spectrometer working at X-band frequencies around 9.2 GHz. The external magnetic field $B_{0}$ can be varied up to $2.56 \mathrm{~T}$. For the PM-FMR technique a light-access cylindrical $\mathrm{TE}_{011}$ microwave cavity is used. This allows a $21 \mathrm{~mW}$ HeNe laser beam to be focussed onto the sample which is placed in the centre of the cavity with the [110]-direction being parallel to the external magnetic field $B_{0}$. The lateral extent of the area where the microwave resonance absorption $P_{a b s}$ is modified by the thermal wave is determined by the diameter of the focal spot $(20 \mu \mathrm{m})$ and the modulation frequency $\left(v_{\text {mod }}\right)$ dependent thermal diffusion length of the sample $(3 \mu \mathrm{m}$ at $v_{\text {mod }}=800 \mathrm{kHz}$ ). Even for the highest modulation frequencies used in the experiments shown here, the sample is thermally thin $\left(1.7 \mu \mathrm{m}\right.$ at $\left.v_{\text {mod }}=2.5 \mathrm{MHz}\right)$. Therefore, no depth resolved information is expected when changing $v_{\text {mod }}$ within the accessible frequency range. The PM-FMR signal is detected with the electronic circuitry of the conventional spectrometer replacing the field modulation of conventional FMR with the temperature modulation due to the localized laser heating. For a more detailed description of the experimental setup the reader is referred to $[10,8]$.

The measurements were carried out on a $20 \mathrm{~nm}$ thick, $3 \mathrm{~mm} \varnothing(001)$ Fe film epitaxially grown on GaAs substrate. For details of the sample preparation and the sample structure see Ref. [2,4]. PM-FMR spectra at $v_{\text {mod }}=800 \mathrm{kHz}$ have been recorded with the laser beam scanning 18 by 18 positions on a grid of $2.8 \times$ $2.88 \mathrm{~mm}^{2}$. As the inhomogeneities to be detected can be observed on a larger scale than the one defined by the spatial resolution of $30 \mu \mathrm{m}$ the step width has been chosen large compared to this value in order to reduce the measurement time.

\section{RESULTS AND DISCUSSION}

Fig. 1a shows the magnetic part of a typical PM-FMR spectrum obtained in the configuration described above which exhibits two resonance lines. The line at the smaller value of $B_{0}$ corresponds to the case where the magnetization $M$ precesses around the direction of an internal effective magnetic field which is not aligned parallel to the external field $B_{0}$ (not aligned case). The line at higher field is due to a precession of the magnetization $M$ around a direction close to the external field $B_{0}$ (aligned case). The equilibrium angles of the magnetization $M$ were determined by angle dependent conventional FMR measurements $[4,12]$. Theoretical considerations [12] show that the spatial distribution of the magnetization $M$ and that of the first order crystalline anisotropy constant $K_{I}$ can be self-consistently determined from the resonance line positions. For comparison, in Fig. la the conventional integral FMR signal is also displayed.

Whereas the conventional field modulation FMR signal is proportional to the field derivative of the imaginary part of the high-frequency susceptibility $\chi^{\prime \prime}$, in the PM-FMR technique the field modulation is replaced by a temperature modulation. As $\chi^{\prime \prime}(T)=\chi^{\prime \prime}\left(M(T), K_{l}(T), \ldots\right)$ is a function of several temperature dependent quantities, using the lock-in technique one measures the first Fourier component of $\chi^{\prime \prime}$ weighted with the temperature distribution inside the sample $(s)$ thus resulting in a lateral resolution [8]:

$$
S_{P M}=\int_{s} \frac{\partial \chi^{\prime \prime}}{\partial T} \vartheta_{s} d \vec{r}
$$

In the case of the investigated epitaxial Fe-film the temperature dependence of $K_{I}$ yields the main contribution to $S_{P M}$. As the temperature coefficient of this quantity is negative, the PM-FMR lines as a function of $B_{0}$ start with negative slopes first. Frequency dependent PM measurements have been conducted from 
$10 \mathrm{~Hz}$ to $2.5 \mathrm{MHz}$. In the whole range, the signal exhibits a $\omega^{-1 / 2}$ dependence as expected for a thermally thin sample.

Figs. 1c and 1d display the lateral variation for $M$ and $K_{l}$ obtained from the PM-FMR mapping. Fig. 1b shows the lateral dependence of the background signal which is attributed to the electronic properties of the semiconducting substrate [13]. A comparison of these images indicate large deviations in the electric and magnetic parameters within an area of approx. $640 \times 800 \mu \mathrm{m}^{2}$ near the coordinates $x=0 \mu \mathrm{m}, y=$ $-1440 \mu \mathrm{m}$. However, the maximum deviations of the magnetic parameters from their respective mean values in that area are quite small $\left( \pm 3.0 \%\right.$ for $M ; \pm 3.8 \%$ for $\left.K_{l}\right)$ demonstrating the high sensitivity of the technique. In the remaining part of the sample only minor changes are observed which are less than $1 \%$ for both $M$ and $K_{l}$. The experimental uncertainties of these magnetic parameters are of the same order or magnitude.
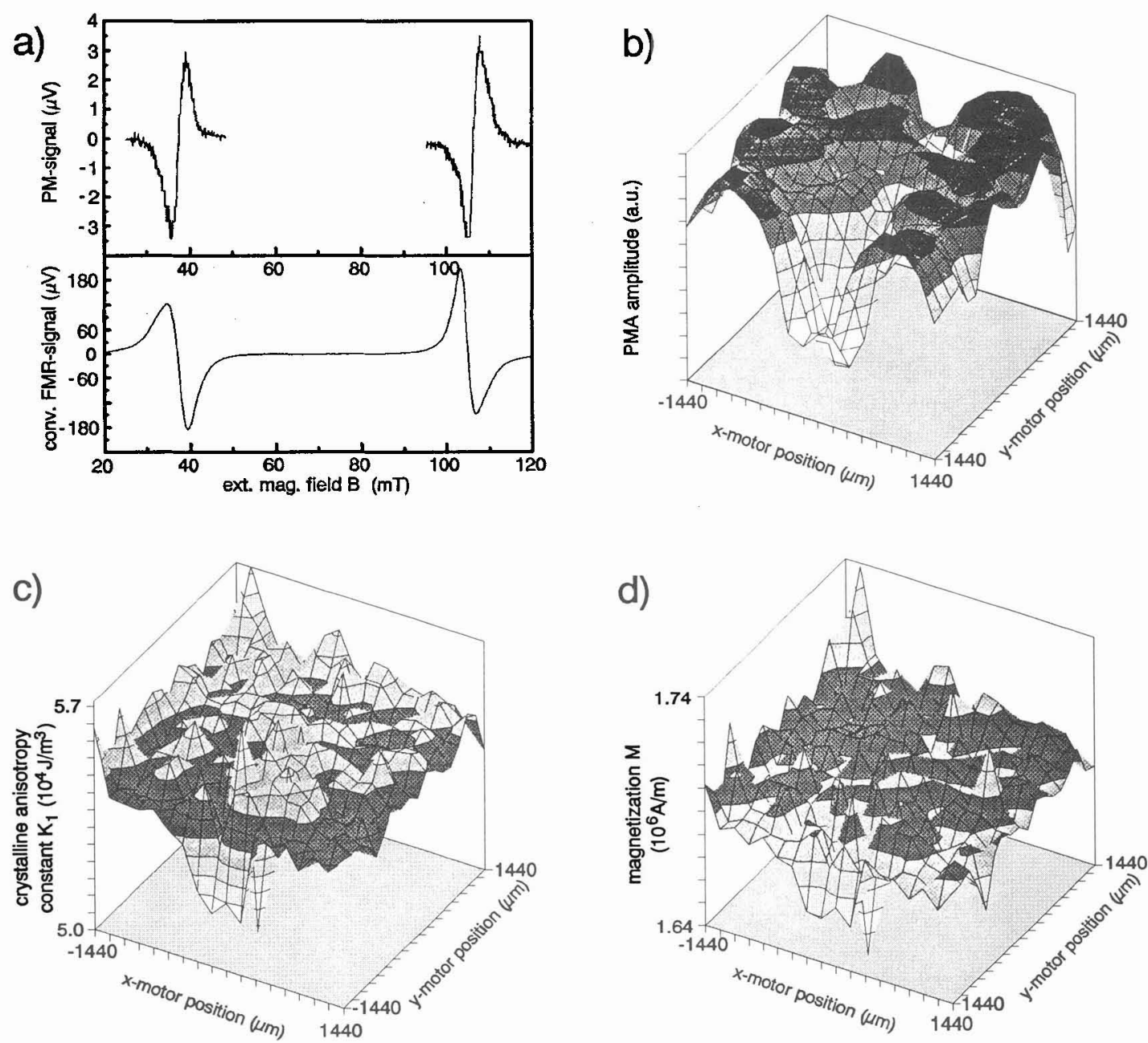

Fig. 1. (a) PM-FMR and conventional FMR spectra of a $20 \mathrm{~nm} \mathrm{Fe}(001)$ film epitaxially grown on GaAs. The magnetic field $B_{0}$ was parallel to the [110] crystal direction. The PM data were corrected for the influence of the semiconductor substrate. (b) Logarithmic plot of background signal due to GaAs substrate. (c,d) Spatial variation of the crystalline anisotropy constant $K_{1}$ and the magnetization $M$ calculated from the line positions of spatially resolved PM-FMR spectra. 


\section{CONCLUSIONS}

In this paper the PM-FMR technique has been successfully applied to the local detection of FMR in thin epitaxial Fe films. For the first time, an imaging of the magnetization $M$ and the crystalline anisotropy constant $K_{1}$ is presented. The spatially resolved results, not accessible to the conventional FMR technique, confirm the high quality of our samples. The averaged values of the PM data are consistent with the values obtained from an extensive conventional angle dependent FMR study on the same sample, published somewhere else [12].

\section{Acknowledgements}

The authors are indebted to P. Grünberg and J. A. Wolf (KFA Jülich, IFF 4) for preparing the samples. This work is supported by the Deutsche Forschungsgemeinschaft, SFB 166.

\section{References.}

[1] M. Vohl et al., J. Appl. Phys. 69 (1991) 4802- 4807

[2] J.A. Wolf, Strukturelle Gesichtspunkte der magnetischen Zwischenschichtkopplung im $\mathrm{Fe} / \mathrm{Cr} / \mathrm{Fe}$ System, $\mathrm{PhD}$ thesis D38 Univ. Köln, published as Berichte des Forschungszentrums Jülich; JÜL2743, March 1993.

[3] J. F. Cochran, J. M. Rudd, M. From, B. Heinrich, W. Bennett, W. Schwarzacher, and W. F. Egelhoff, Jr, Phys. Rev. B 45 (1992) 4676-4685

[4] E. C. da Silva, R. Meckenstock, O. von Geisau, R. Kordecki, J. Pelzl, J. A. Wolf, and P. Grünberg, J. Magn. Magn. Mat. 121 (1993) 528-531.

[5] G. V. Skrotskii and L. V. Kurbatov, in Ferromagnetic Resonance, S. V. Vonsovskii, Ed. (Pergamon Press, Oxford, 1966), pp. 12-77.

[6] Th. Orth, U. Netzelmann, and J. Pelzl, Appl. Phys. Lett. 53 (1988) 1979-1981.

[7] J. Pelzl, U. Netzelmann, Th. Orth, and R. Kordecki, in Photoaconstic and Photothermal Phenomena II, J. C. Murphy et al., Eds., (Springer Verlag, Berlin Heidelberg, 1990), pp. 2-12.

[8] J. Pelzl and O. von Geisau, in Non Destructive Evaluation, A. Mandelis, Ed., (Prentice Hall, Englewood Cliffs, 1994), pp. 237-320.

[9] J. Pelzl and U. Netzelmann, in Photoacoustic, Photothermal and Photochemical Processes at Surfaces and in Thin Films, P. Hess, Ed., (Springer Verlag, Berlin, 1989), pp. 313-365.

[10] $O$. von Geisau, Photothermische Untersuchungen der FMR langwelliger kollektiver magnetischer Anregungen in YIG, PhD-Thesis, Ruhr-Universität Bochum, 1993

[11] O. von Geisau, F. Rödelsperger, F. Schreiber, H. Benner, and J. Pelzl, paper HO-04 8ITMP3

[12] R. Meckenstock, O. von Geisau, F. Schreiber, J. A. Wolf, and J. Pelzl, submitted to J. Appl Phys.

[13] O. von Geisau, R. Meckenstock, F. Schreiber, and J. Pelzl, paper CO-01 8ITMP3 